\title{
Key Learnings from Twenty Years of Neural Network Applications in the Chemical Industry
}

\author{
Aaron J. Owens \\ DuPont Company, Engineering Research and Technology, \\ POB 80356, Wilmington, DE, 19880-0356, U.S.A. \\ aaron.j.owens@usa.dupont.com
}

\begin{abstract}
This talk summarizes several points that have been learned about applying Artificial Neural Networks in the chemical industry. Artificial Neural Networks are one of the major tools of Empirical Process Modeling, but not the only one. To properly assess the appropriate model complexity, combine information about both the Training and the Test data sets. A neural network, or any other empirical model, is better at making predictions than the comparison between modeled and observed data shows. Finally, it is important to exploit synergies with other disciplines and practitioners to stimulate the use of Neural Networks in industry.
\end{abstract}

Keywords: Artificial Neural Networks, Neural Network Applications, Stopping Criteria, Empirical Model Validity.

\section{Introduction}

DuPont has had a long history of supporting neural network applications in the chemical industry. Initial efforts were aimed at commercial applications in digital image processing during the 1980s. In 1990, the Neural Network Resource Center was established to catalyze the use of artificial neural network models, particularly the Back Propagation Network [1], [2], internally throughout the Company. The author has personally been involved in this these efforts ever since, except for a brief, misguided detour into management. This talk summarizes some of the "key learnings" about neural network applications resulting from those efforts.

\section{Neural Networks as an Empirical Process Modeling Tool}

Empirical Process Modeling is data driven, rather than emphasizing fundamental equations. It can be described colloquially as Modeling a Spreadsheet. The spreadsheet metaphor has been useful in helping our internal practitioners to decide what type of model to use. The Process being modeled can be of any kind: chemical, physical, biological, business, financial, marketing, etc. DuPont is a large, international Science company. Our Empirical Process Modeling efforts have spanned many products, functions, and regions. 


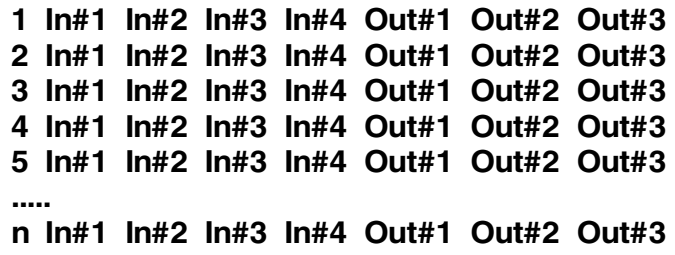

1 In\#1 In\#2 In\#3 In\#4 Out\#1 Out\#2 Out\#3

2 In\#1 In\#2 In\#3 In\#4 Out\#1 Out\#2 Out\#3

3 In\#1 In\#2 In\#3 In\#4 Out\#1 Out\#2 Out\#3

4 In\#1 In\#2 In\#3 In\#4 Out\#1 Out\#2 Out\#3

5 In\#1 In\#2 In\#3 In\#4 Out\#1 Out\#2 Out\#3

n In\#1 In\#2 In\#3 In\#4 Out\#1 Out\#2 Out\#3

\section{SPREADSHEET}

\section{EMPIRICAL MODEL}

Fig. 1. The Spreadsheet Metaphor. In Empirical Modeling, data are arranged into a Spreadsheet with real-valued Inputs and Outputs from the process being modeled.

DuPont's proprietary neural network software, called the Neural WebKit, uses a simple spreadsheet-based data format. Each Exemplar or case appears on a row. It consists of the following columns: a unique integer Exemplar Number followed by the numerical values of all of the process Inputs $X$ and then of all the process Outputs $Y$. See Figure 1.

The empirical modeling tool, here illustrated by the Back Propagation Network (BPN), is applied to the data set to obtain the parameters for an Empirical Model. The goal of Empirical Modeling is to maximize accuracy of the prediction of the Ys from the Xs.

The software requires that the data set be pre-processed so that there are no missing entries and that all variables are real-valued. Categorical variables need to be unary encoded, with the Machines A, B, or C coded as the three variables [ [ $\left.\begin{array}{lll}1 & 0 & 0\end{array}\right],\left[\begin{array}{lll}0 & 1 & 0\end{array}\right]$ and $\left[\begin{array}{lll}0 & 0 & 1\end{array}\right]$.

The appropriate modeling technique to use in building the empirical model is determined by the shape of the spreadsheet, as illustrated in Figure 2, along with the data's Signal to Noise ratio. For Short and Fat spreadsheets, there are fewer (often many fewer) exemplars than variables, so a sub-linear method is necessary. To identify the model coefficients, the number of model parameters needs to be less than the number of observations. Even simple Multiple Linear Regression (MLR) cannot be used for these cases.

For Short and Fat spreadsheets, the recommended Chemometric technique is Partial Least Squares (PLS) [3]. Classical statistical techniques (like MLR and polynomial regression) deal with cases in which the spreadsheet matrix is close to Square. In fact, classical statistical Design of Experiments (DOE) minimizes the number of experiments so that it is just a few more than the number of parameters to be estimated, so the spreadsheet is nearly Square. 


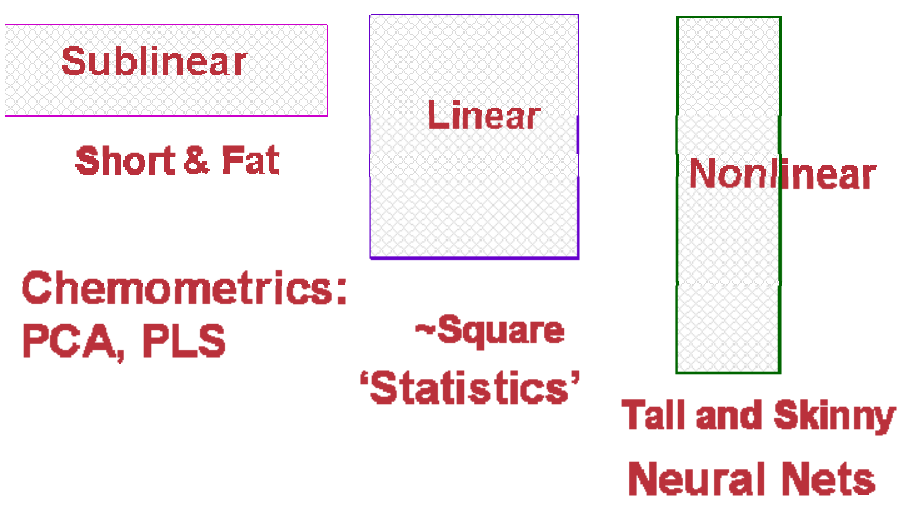

Fig. 2. What shape is your spreadsheet? Chemometrics techniques like Partial Least Squares [PLS] are best suited to Short and Fat matrices. Classical statistical techniques are appropriate with Square matrices. Neural network can be applied to Tall and Skinny matrices. For low Signal to Noise data sets, more Exemplars are required to accurately determine model parameters.

Neural networks are more appropriate only in the relatively data-rich case of a Tall and Skinny data matrix. Then there are enough observations to allow the system to identify a model with more coefficients than Inputs [4], [5].

There are a couple of caveats with respect to selecting the appropriate model. First, while a matrix may appear Tall and Skinny, if the underlying process has a small Signal to Noise ratio (i.e., it is dominated by noise), then more -- perhaps many more - observations are required to accurately identify the model parameters. So it may be appropriate to use PLS for a Tall and Skinny matrix with low Signal to Noise. Second, both PLS and neural networks can successfully model linear systems. So in Empirical Modeling the classical statistical tools are actually seldom needed, as long as the objective of the model is accurate Prediction. While PLS and neural networks have tools to assess variable importance, only classical statistical techniques allow you -for example -- to point to a specific coefficient as the cross term between variables $\mathrm{x}(3)$ and $\mathrm{x}(4)$, as well as give its statistical uncertainty.

Most of the time needed to prepare an Empirical Process Model comes from collecting and checking the data. Once the data are in a Spreadsheet, the time to do an analysis is usually trivial, especially with PLS. So it is recommended to always run a PLS analysis before proceeding to use a neural network on the same data set. Note that you can transform a single hidden layer Back Propagation Network to the PLS structure simply by eliminating the squashing functions. In addition, commercial PLS software has good graphical tools for interpreting the models with multiple-input, multiple-output mappings.

\section{Use Both Training and Test Data to Find Model Complexity}

More than $90 \%$ of the neural network applications at DuPont have used the Back Propagation Network with a single hidden layer [6]. That methodology has remained 
basically unchanged for more than twenty years. There are some special circumstances in which more hidden layers are used, often a Bottleneck layer [7], but they are limited. No matter how many hidden layers are used, there is usually one called the Mapping Layer, which controls the complexity of the input-output mapping. A small number of nodes in the Mapping layer corresponds to low model complexity, and the representational ability of the neural network grows as that number is increased.

Because training a neural network can be time-consuming, it is usual to use a single Train/Test data split, rather than more sophisticated multiple cross-validation methods from the Chemometrics literature. For neural networks, the data are split, either randomly or by design, into a Training data set and a Test data set. Models are developed ('trained') on the Training data, and their expected performance for generalization is estimated from the Test data. If enough data are available, a separate Validation data set is sometimes used as a final check on the accuracy of the system.

\subsection{Three Train/Test Methods: Scattergun, Train and Grow, and Early Stopping}

There are at least three competing methods for developing neural network models of the appropriate complexity. In the Scattergun method, neural networks with 1 through $\mathrm{M}$ hidden nodes in the Mapping layer are randomly initialized. They are all trained to completion, and the choice is the one with the minimum Test error. Because it uses multiple random initializations, this method is not robust.

A method with wide application in DuPont is Train and Grow [6]. Start with one node in the Mapping Layer, and train the model on the Training data to completion. Note the performance on the Test data. Grow the trained network by adding a second mapping node, with small random values for the new connection weights, and then train it. Continue training and growing until the Test error increases with increasing nodes. This method gives a more robust and reproducible Train/Test path, because the models usually stay in the same basin of attraction in weight space.

Early Stopping is a viable alternative to Train and Grow, particularly if modeling similar systems has given you a reasonable starting point. In this method, a single, large number of mapping units is selected, more than are actually needed. The weights are randomly initialized and then training begins. Rather than training to completion, the Root Mean Square (RMS) Errors of the Train and Test data sets are monitored as a function of the number of training iterations. Select the Number of Training Iterations giving the minimum RMS Test error as the appropriate model complexity [8].

Note that with Early Stopping, training is stopped before the weights reach their final optimum values. This method runs much more quickly than Train and Grow, since only one model is developed. But Early Stopping is more sensitive to initial conditions, particularly for multiple-hidden-layer models.

For all of these methods, the important piece of information is the minimum RMS Test error, since that should predict performance on new data. As a final step, it is always recommended to re-train the model using All of the data and stopping when the appropriate $R M S$ Test error limit is reached. 
Historically at DuPont, the Shotgun method was first tried but quickly abandoned as too unreliable. Train and Grow continues to be used for most applications with a single hidden layer [6]. For multiple-hidden-layer Bottleneck Neural Networks, Early Stopping has been adopted for decreased training time [8].

\subsection{A Complexity Criterion Using Both Train and Test Errors}

To select the best model, the neural network community has heavily weighted one criterion - minimum RMS Test error - but ignored some additional relevant information. A good model would be one that has a clear minimum in the RMS Test error but also has Train and Test errors similar to each other. In other words, we should also consider the RMS Training error in selecting the appropriate model complexity. If it is significantly less than (or greater than) the RMS Test error at its minimum (as a function of Number of Hidden Units for Train and Grow or Number of Iterations for Early Stopping), that indicates a modeling failure. The Train and Test data sets are, for some reason, not compatible.

We propose a new metric that takes into account both RMS Train and Test errors. It optimizes both the Test error and the difference between Train and Test. An equation that accomplished this is to minimize

$$
\alpha^{*}(\text { RMS Test error })+(1-\alpha) * \mid \text { RMS Train error - RMS Test Errorl . }
$$

If $\alpha=1$, only the RMS Test error matters (as has been conventional). If $\alpha=0$, the criterion assures that the RMS Train and Test errors are the same. After looking at dozens of cases, we propose $\alpha=0.5$ as a good compromise. That is a 50/50 combination of the two parts of Equation (1).

The process is to enter the RMS Train and RMS Test errors, along with the corresponding Number of Hidden Units or Iteration Number, into a spreadsheet. The spreadsheet locates the minimum of the function of Equation (1) and records the appropriate Model Complexity at that point.

See the example in Figure 3 below. The appropriate model complexity (Number of Iterations) is selected by minimizing the RMS Test error (plus symbols) and the absolute difference between RMS Train error (filled circles) and RMS Test error. A table with the plotted values, for this hypothetical example, appears on the right, where Train and Test refer to scaled RMS errors and Crit. is the new criterion of Equation (1) with $\alpha=0.5$. The solid curve shows this new criterion.

The usual procedure, considering only the minimum RMS Test error, gives an optimum at Iteration 10 (underlined), where the Train and Test errors are 0.0071 and 0.0264 , respectively (differing by a factor of 3.7!). The New Criterion selects 8 Iterations (bold and underlined), where the train and Test errors are 0.0139 and 0.0285 (a smaller factor of 2.1).

Adopting this New Criterion should give a more balanced selection of the appropriate model complexity. Incidentally, it works well for PLS models too, where the Train and Test errors are replaced by Calibration and Cross-Validation errors, and the complexity parameter is the Number of Latent Variables selected.

An important final step is to re-train the neural network model using All of the data (Training + Test data sets), stopping at the appropriate Model Complexity: the 
selected optimum Number of Hidden Units for Train and Grow or the Number of Iterations for Early Stopping.

\section{An Empirical Model Is Better Than the Data Show}

The discussion in this section may be well known to statisticians, but many people in the neural network and chemometrics communities may not be aware of it. Quite simply, any empirical model fit by least squares has its model coefficients and hence its prediction accuracy improved with additional data from the same process. However, by looking at model predictions compared with just the (noisy) data from that process itself, you cannot prove this.

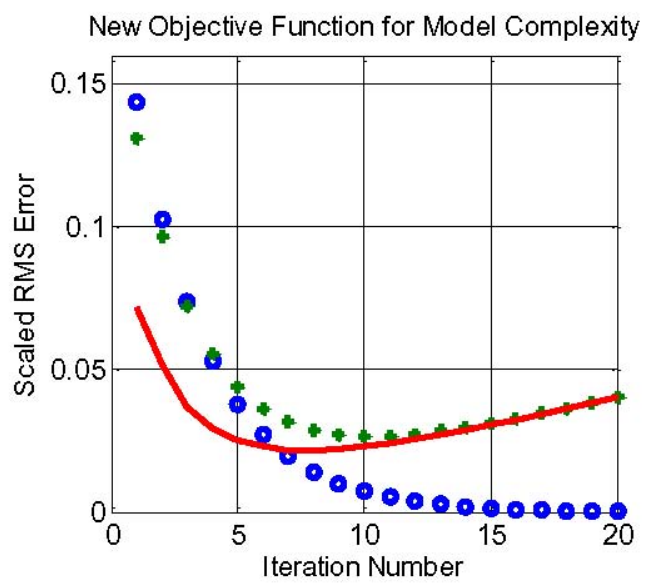

\begin{tabular}{|c|c|c|c|}
\hline \multicolumn{2}{|c|}{ Iters Trai } & Test & Crit. \\
\hline 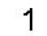 & 0.1433 & 0.1310 & 0.0717 \\
\hline 2 & 0.1027 & 0.0964 & 0.0513 \\
\hline 3 & 0.0736 & 0.0722 & 0.0368 \\
\hline 4 & 0.0527 & 0.0554 & 0.0291 \\
\hline 5 & 0.0378 & 0.0440 & 0.0251 \\
\hline 6 & 0.0271 & 0.0364 & 0.0228 \\
\hline 7 & 0.0194 & 0.0315 & 0.0218 \\
\hline 8 & 0.0139 & 0.0285 & 0.0216 \\
\hline 9 & 0.0100 & 0.0270 & 0.0220 \\
\hline 10 & 0.0071 & 0.0264 & 0.0229 \\
\hline 11 & 0.0051 & 0.0266 & 0.0240 \\
\hline 12 & 0.0037 & 0.0273 & 0.0255 \\
\hline 13 & 0.0026 & 0.0284 & 0.0270 \\
\hline 14 & 0.0019 & 0.0297 & 0.0288 \\
\hline 15 & 0.0013 & 0.0312 & 0.0305 \\
\hline 16 & 0.0010 & 0.0329 & 0.0324 \\
\hline 17 & 0.0007 & 0.0346 & 0.0343 \\
\hline 18 & 0.0005 & 0.0364 & 0.0362 \\
\hline 19 & 0.0004 & 0.03 & 0.0381 \\
\hline 20 & 0.0003 & & \\
\hline
\end{tabular}

Fig. 3. New Objective Function for Selecting Model Complexity

Suppose that there is a Process with multivariate Inputs $\mathrm{X}$ and Outputs $\mathrm{Y}$. There will also be noise $(\varepsilon)$ in the process. A model for this kind of process is

$$
Y_{\mathrm{k}}=\mathrm{f}\left(X_{\mathrm{k}}\right)+\varepsilon_{\mathrm{k}}
$$

Here the subscript k refers to the kth Exemplar.

The function $\mathrm{f}\left(X_{\mathrm{k}}\right)$ is the True Relationship between $X$ and $Y$, but each observation or Exemplar has an associated noise $(\varepsilon)$. As is customary, the random errors $\varepsilon$ for the exemplars are assumed to be Independent and Identically Distributed (IID), sampled from a Normal distribution with mean 0 and standard deviation $\sigma$.

In a neural network, the True Relationship is approximated by a neural network model $\mathrm{f}^{\mathrm{NN}}(X)$ with many parameters. After the training of neural network to get the 
optimum values of the model parameters, we assume that the neural network model approximates the truth, or:

$$
\mathrm{f}^{\mathrm{NN}}(X) \sim \mathrm{f}(X) .
$$

Note that even if Equation (3) represents full equality, when we use $\mathrm{f}^{\mathrm{NN}}(X)$ to estimate the (noisy) observed value of $Y$, we see from Equation (2) that the difference between predicted and observed is equal to the noise (with standard deviation $\sigma$ ). While the model can get very accurate, as more and more data points are added, when we compare it with the noisy real data we still get a Root Mean Square error of $\sigma$.

\subsection{Simple Linear Example: Numerical Simulations}

The effect can be illustrated with a very simple example, with univariate $\mathrm{x}$ and $\mathrm{y}$ related by a linear relationship. If Equation (3) is applicable (i.e., the neural network, when trained, approaches the true X-Y noise-free relationship), the same kind of results would be obtained with a Neural Network model rather than this linear model. The linear results are easier to explain.

Equation (2) with this underlying model becomes:

$$
y_{\mathrm{k}}=\mathrm{c} * x_{\mathrm{k}}+\varepsilon_{\mathrm{k}} .
$$

Equation (4) is used to generate the Observed data. For specificity, take c $=6$ and $\sigma=1$.

Numerical simulations were performed using a simple Matlab script. Each of $\mathrm{N}$ Exemplars has $x$ values between uniformly sampled between 0 and 1, then added random noise with $\sigma=1$ to get Observed $y$ values from Equation (4).

To simulate the model-building phase, a simple linear regression model (Equation $5)$ was fitted to the $\mathrm{N}$ data pairs $(x, y)$.

$$
y=a^{*} x .
$$

The slope $a$ and the RMS error between the Observed and Predicted values of $y$ were recorded for each realization. Calculations were made with the number of

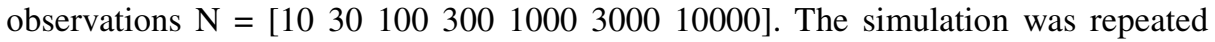
with each value of $\mathrm{N}$ for a total of 1000 iterations and averaged over all 1000 of them to reduce sampling noise.

See the results in Figure 4. The RMS error between the model and the (noisy) Observed data is shown with the symbol ' $\mathrm{x}$ '. The RMS error between model and the underlying True (noise-free) data is shown with the symbol 'o'. The RMS error between the true slope (c =6) and the model-estimated slope (a) is shown with the symbol ' $*$ '. The line is drawn to guide the eye; its equation is $1 /$ sqrt $(\mathrm{N})$, where $\mathrm{N}$ is the Number of Samples.

Note that the RMS error between Predicted and Observed y is close to $\sigma=1$, as discussed above. (It is labeled 'Observed' in the figure.) Increasing $\mathrm{N}$ does not change this estimate, which is roughly constant with $\mathrm{N}$. With added data, the RMS error of the model prediction compared with the (noisy) Observed data does not decrease.

Next consider the value of the modeled slope, $a$. It is close to the true value of $\mathrm{c}=$ 6 for $\mathrm{N}=10$, but it gets increasingly closer, with error varying as $1 /$ sqrt $(\mathrm{N})$, as $\mathrm{N}$ is increased. While standard statistical packages would estimate the error limits on the 
slope, there is no direct way in most PLS or neural network calculations to extract that information. The modeler may not realize that the model is getting more accurate with additional data, especially if RMS error of model predictions versus the observed noisy data is the only evaluation factor.

Finally, since this is a Simulation, one can 'play God' and look at how the model predicted the underlying actual relationship, $\mathrm{y}=6^{*} \mathrm{x}$, ignoring the noise in Equation (4). The points labeled 'True' in Figure 4 shows that the model prediction accuracy, compared with the underlying noise-free generating model, does in fact get better as $1 /$ sort(N). In this simulation, the RMS prediction error from the noisy observed data remains close to $\sigma=1$, while the model's prediction of the true underlying relationship has an RMS error of about 0.01 with 10,000 samples.

So the empirical model predictions actually are much more accurate than a comparison between the predicted and observed data show. The law of large numbers applies here, so the model prediction error of Neural Networks and PLS models really does decrease as $1 / \operatorname{sqrt}(\mathrm{N})$, as in standard statistical model estimation.

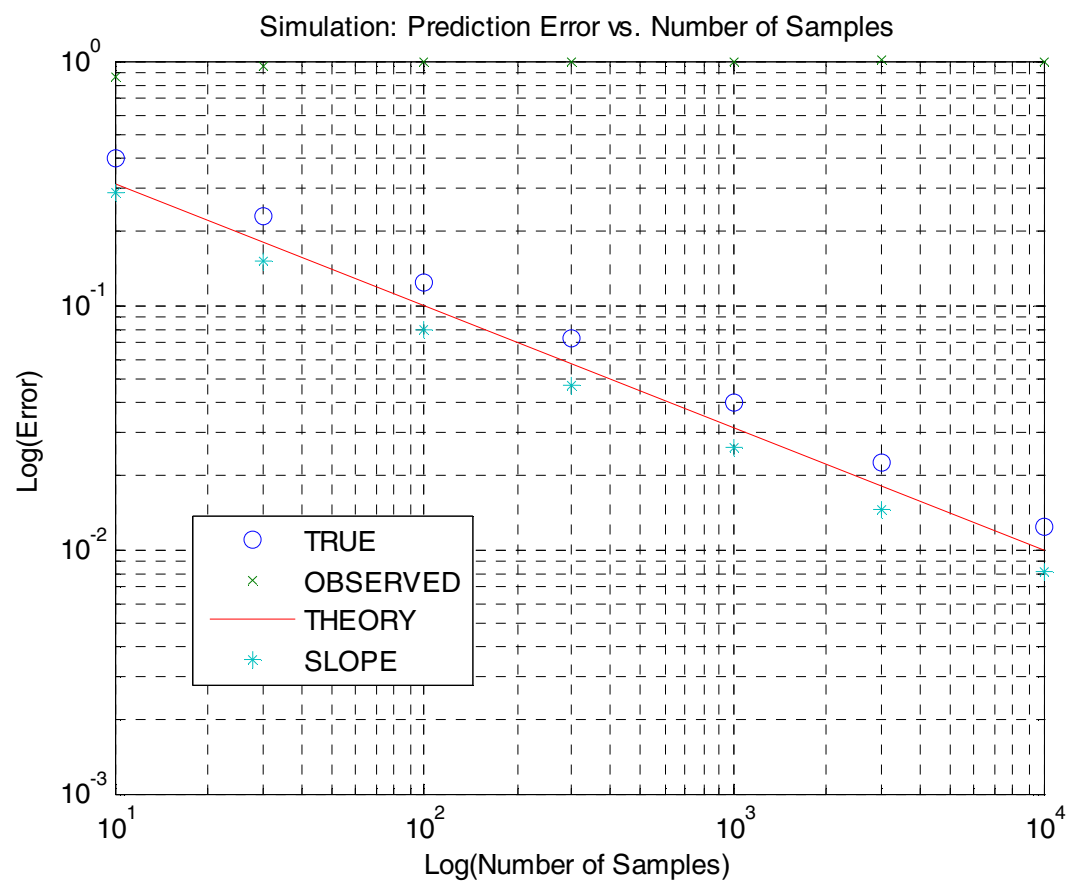

Fig. 4. Summary of Numerical Simulations of Model Accuracy, on a Log-Log Plot

\section{Synergies to Increase the Use of Neural Networks in Industry}

As discussed in Section 2 above, the experience at DuPont has shown Neural Networks are best used as an Empirical Process Modeling tool, in conjunction with traditional statistical analysis and methods from Chemometrics. In light of this, the 
Neural Network practitioner in industry (as well as in research) should not be isolated from their colleagues who are applied statisticians and chemometricians.

Whatever success achieved in introducing Neural Networks at DuPont [9] was catalyzed by neural net modelers interacting with these other groups. As early as 1990, when few statisticians would deign to consider Neural Networks, a close working relationship was established with the Applied Statistics group. Industrial statisticians are very appropriate Neural Network practitioners, because of their understanding of nonlinear function fitting and their appreciation for the effects of uncertainty. The neural network and statistics groups had cross-training sessions about Neural Networks, Exploratory Data Analysis, and Design of Experiments. The internally developed Neural Network software was enhanced by adding Analysis of Variance tables, incorporating Degrees of Freedom thinking into the $\mathrm{r}^{\wedge} 2$ calculations, and surface and contour plots of results. Our group presented one of the first papers on Neural Networks (for process modeling and control) at an American Statistical Association in 1992 [10]. Two statisticians reported on different kinds of crossvalidation for Neural Networks at the same conference the following year [11].

About the same time, Mike Piovoso introduced us to chemometrics and began our interactions with the process control community. We learned how to interpret the Hidden Layer outputs using Principal Component Analysis and recognized the similarity between the structure of a Back Propagation Network (with one hidden layer) and Partial Least Squares (PLS). Effectively, PLS is a BPN without the squashing function. PLS software packages showed us the utility of the Modeled versus Observed plot (Goodness of Fit), and the graphical presentation in PLS's Variable Importance Plot guided our Sensitivity Analysis plot. Interactions with process control engineers prompted the early adoption of Neural Networks as Virtual Sensors for model-based control [12]. Neural Networks were subsequently integrated by the major process control vendors into their software.

More importantly than just improving our Neural Network methodology, the contacts with the statisticians and process control engineers gave a wide variety of novel and important applications.

\section{Summary}

The primary "value add" of Neural Networks is that they are extremely good at extracting complex, nonlinear interactions between process input and outputs, particularly when there are enough observations to fit a model with high signal to noise. There are many and varied applications for that kind of tool in industry [13], [14], [15], [16].

Acknowledgments. DuPont colleagues who have contributed significantly to our neural network technology and applications include Tom Lynch, Alicia Walsh, Dewey Donovan, John van Stekelenborg, Vivek Bhide, and Tony Liu. Supporters in the businesses include Dave Alman, Allan Rodrigues, Mahnaz Mohammadi, Tariq Andrea, John Kinney, Arun Aneja, and John Locke. I learned about Chemometrics from Mike Piovoso, Barry Wise (Eigenvector), and John MacGregor (Prosensus). 


\section{References}

1. Rumelhart, D.E., Hinton, G.E., Williams, R.J.: Learning Internal Representations by Error Propagation. In: Rumelhart, McClelland (eds.) Parallel Distributed Processing. Foundations, vol. 1, pp. 318-335. MIT Press, Cambridge (1986)

2. Hertz, J., Krough, A., Palmer, R.G.: Introduction to the Theory of Neural Computation. Addison-Wesley, New York (1991)

3. Software User Guide, PLS Toolbox for Matlab: Eigenvector Research, Inc., Wenatchee, Washington (2011), http: / /wiki.eigenvector.com

4. Ljung, L., Sjoberg, J.: A System Identification Perspective on Neural Nets. In: Kung, et al. (eds.) Neural Networks for Signal Processing II, pp. 423-435. IEEE, Piscataway (1992)

5. Bishop, C.M.: Neural Networks for Pattern Recognition. Clarendon Press, Oxford (1995)

6. Piovoso, M.J., Owens, A.J.: Sensor Data Analysis Using Artificial Neural Networks. In: Arkun, Ray (eds.) Chemical Process Control CPC IV, pp. 101-118. AIChE, New York (1991)

7. Owens, A.J.: Using Fundamental Knowledge to Guide Empirical Model Structure. In: International Conference on Engineering Applications of Neural Networks (EANN 2001), Cagliari (2001)

8. Owens, A.J.: Neural Network Based Product Formulation Models: Early Stopping Saves Training Time. In: International Conference on Engineering Applications of Neural Networks (EANN 2007), Stockholm (2007)

9. Owens, A.J.: Neural Network Based Empirical Modeling in the Chemical Industry. In: International Conference on Engineering Applications of Neural Networks (EANN 1998), Gibraltar (1998)

10. Owens, A.J.: Artificial Neural Networks for Process Modeling and Control. In: Proceedings of the Annual Joint Statistical Meetings of the American Statistical Association, Boston (1992)

11. Bhide, V., Banerjee, A.: Bootstrap Estimates in Neural Network Models. In: Proceedings of the Annual Joint Statistical Meetings of the American Statistical Association, San Francisco (1993)

12. Schnelle Jr., P.D., Fletcher, J.A.: Using Neural Network Based Process Modeling in Process Control. In: Proceedings of the I.S.A./90 International Conference and Exhibition (1990)

13. Hu, Y.H., Hwang, J.-N. (eds.): Handbook of Neural Network Signal Processing. CRC Press, Boca Raton (2001)

14. Badiru, A.B., Cheung, J.Y.: Fuzzy Engineering Expert Systems with Neural Network Applications. Wiley-Interscience, New York (2002)

15. MacKay, D.J.C.: Information Theory, Inference and Learning Algorithms. Cambridge University Press, Cambridge (2003)

16. Suzuki, K. (ed.): Artificial Neural Networks - Methodological Advances and Biomedical Applications. InTech, Rijeka (2011) 